\title{
Hierarchical Bimetallic Ni-Co-P Microflowers with Ultrathin Nanosheet Arrays for Efficient Hydrogen Evolution Reaction over All pH Values
}

Xupo Liu ${ }^{\mathrm{a}}$, Shaofeng Denga, Dongdong Xiao ${ }^{\mathrm{b}}$, Mingxing Gonga ${ }^{\mathrm{a}}$, Jianing Liang ${ }^{\mathrm{a}}$, Yun $\mathrm{Lu}^{\mathrm{a}}$, Tonghui Zhao ${ }^{\text {a }}$, Tao Shen ${ }^{\mathrm{a}}$, Deli Wang ${ }^{\mathrm{a}, *}$

${ }^{a}$ Key Laboratory of Material Chemistry for Energy Conversion and Storage (Huazhong University of Science and Technology), Ministry of Education, Hubei Key Laboratory of Material Chemistry and Service Failure, School of Chemistry and Chemical Engineering, Huazhong University of Science and Technology, Wuhan, 430074, P.R. China.

${ }^{b}$ Beijing National Laboratory for Condensed Matter Physics, Institute of Physics, Chinese Academy of Sciences, Beijing, 100190, P.R. China.

E-mail: wangd181125@hust.edu.cn.
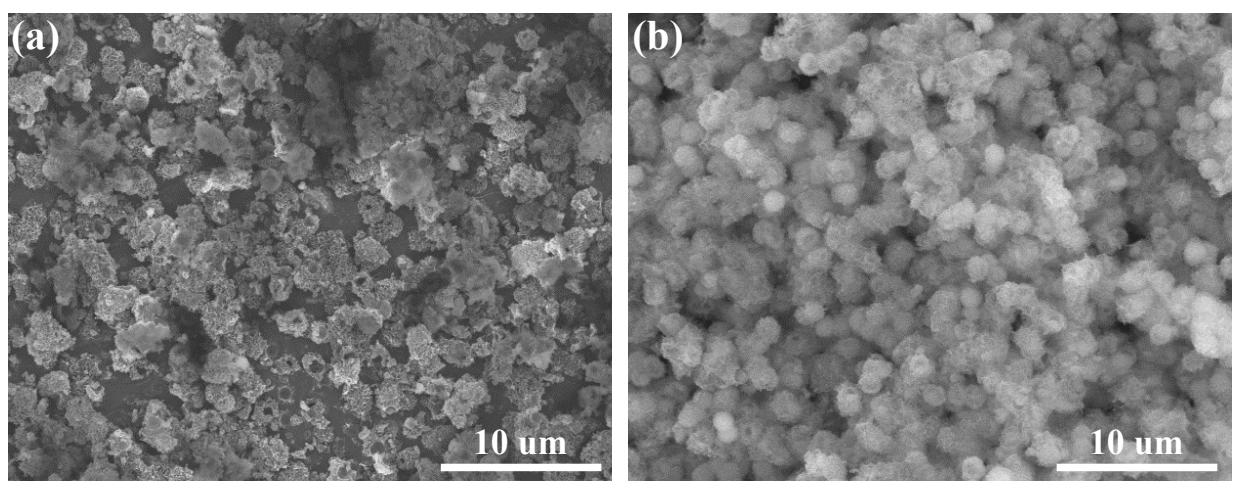

Figure S1 SEM images of (a) Co-G-H and (b) Ni-Co-G-H. 


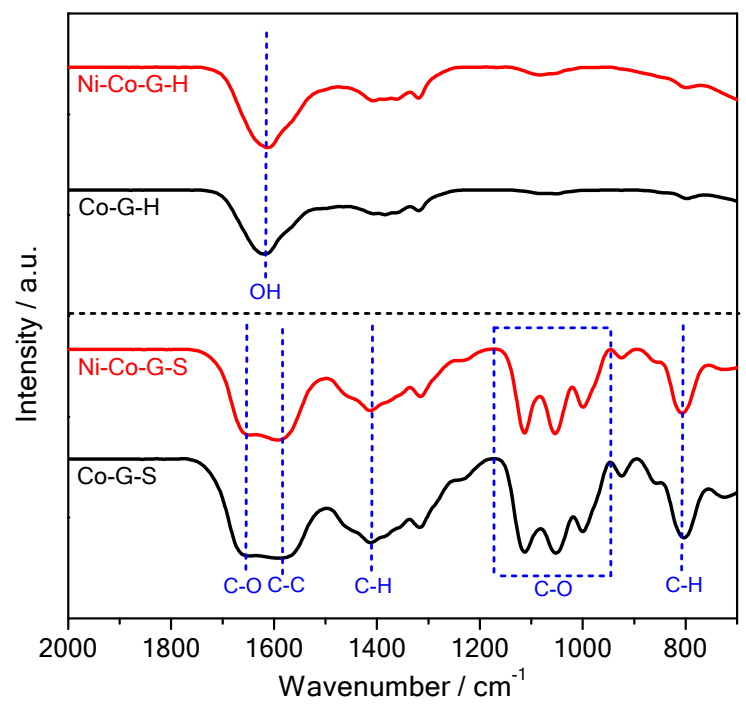

Figure S2 FTIR spectra of Co-G-S, Ni-Co-G-S, Co-G-H and Ni-Co-G-H.
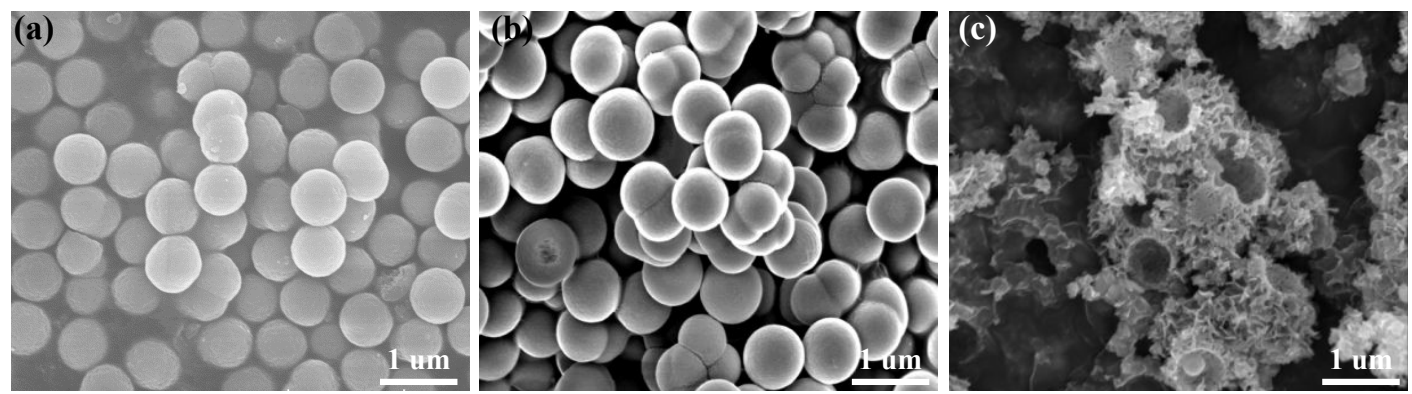

Figure S3 SEM images of (a) Co-P-S, (b) Ni-Co-P-S and (c) Co-P-H.

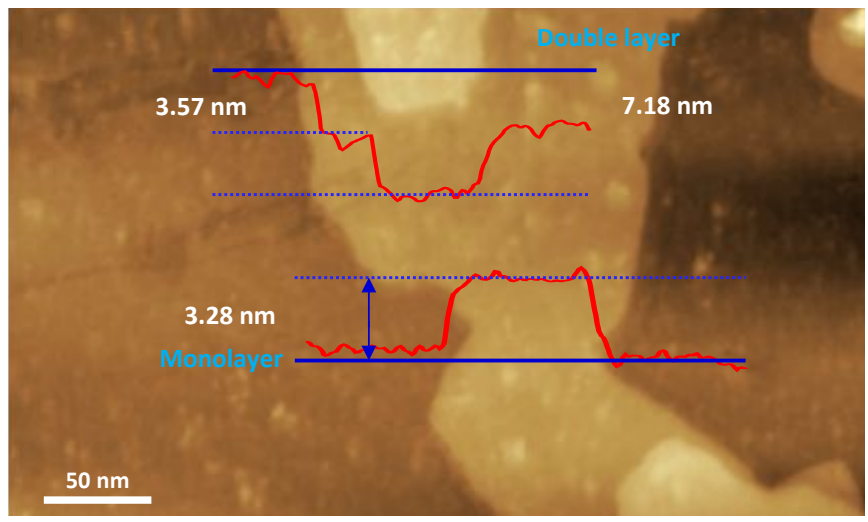

Figure S4 AFM image of nanosheets for Ni-Co-P-H: (up) the double layer with the thickness of $7.18 \mathrm{~nm}$, (down) the monolayer with the thickness of $3.28 \mathrm{~nm}$. The AFM result suggests the Ni-Co-P-H catalyst contains ultrathin nanosheets. Generally, the thickness of ultrathin two-dimensional nanomaterials is typically less than $5 \mathrm{~nm}^{1}$. 

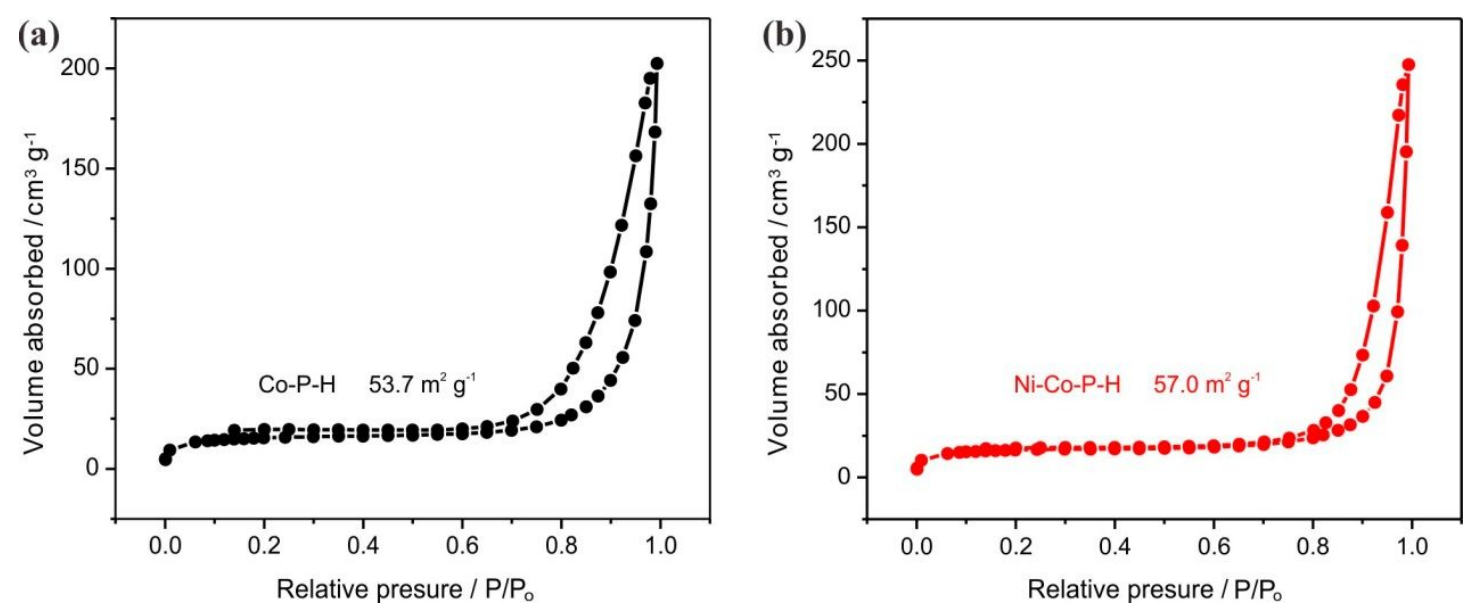

Figure S5 N2 adsorption-desorption isotherms of (a) Co-P-H and (b) Ni-Co-P-H.

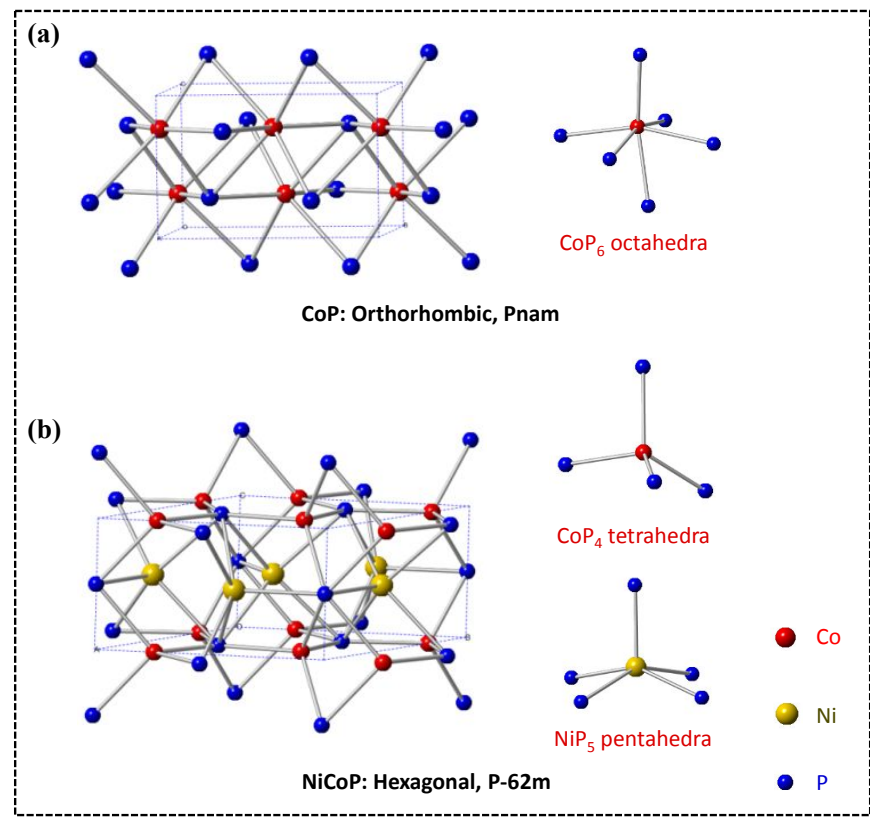

Figure S6 Crystal structures of (a) orthorhombic $\mathrm{CoP}$ and (b) hexagonal NiCoP, unit cells are depicted as dashed blue lines. 

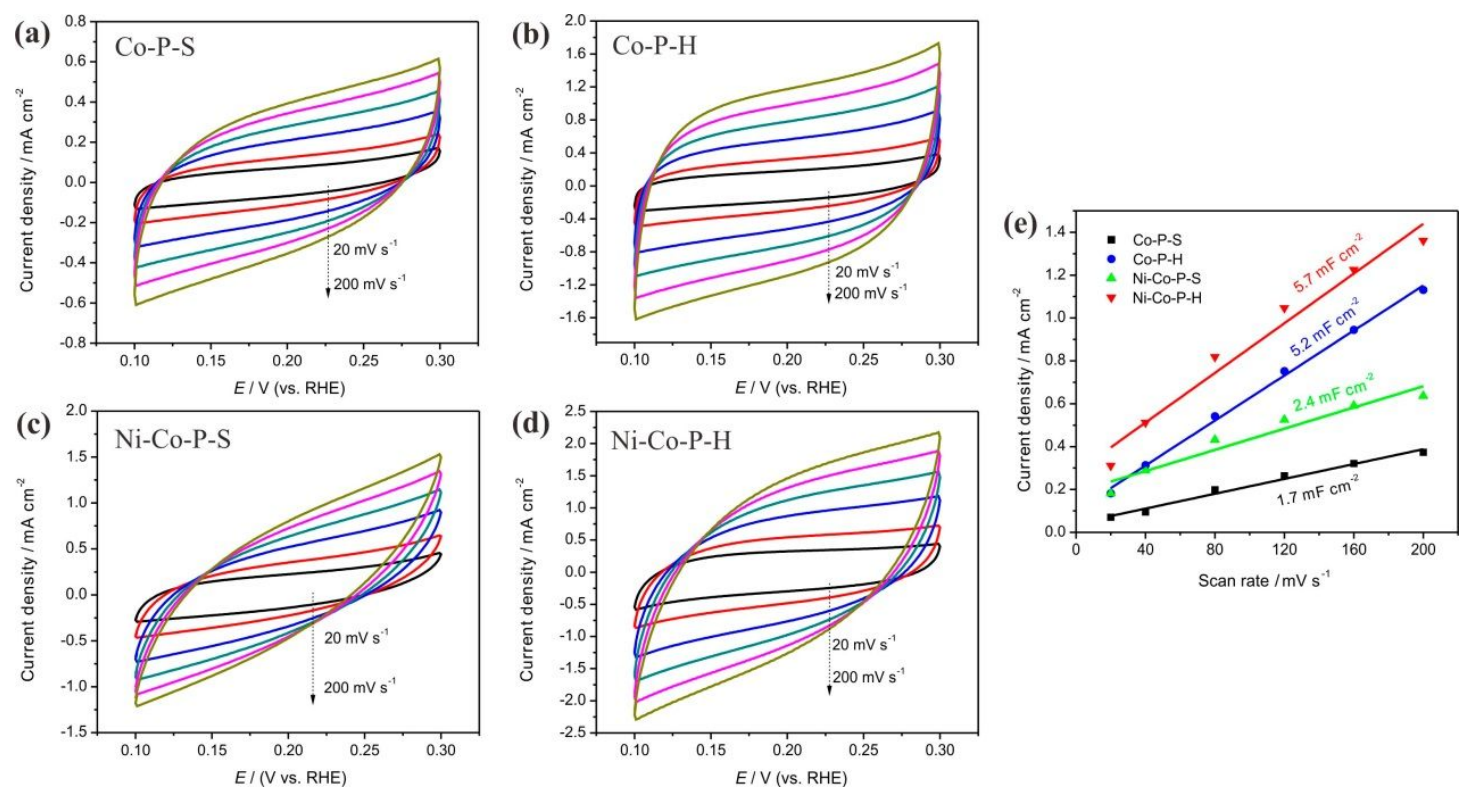

Figure S7 CV curves in the range of $0.1-0.3 \mathrm{~V}$ at various scan rates in $0.5 \mathrm{M} \mathrm{H}_{2} \mathrm{SO}_{4}$ solution: (a) Co-P-S, (b) Co-P-H, (c) Ni-Co-P-S and (d) Ni-Co-P-H; (e) the calculated capacitive currents depending on a function of scan rate.
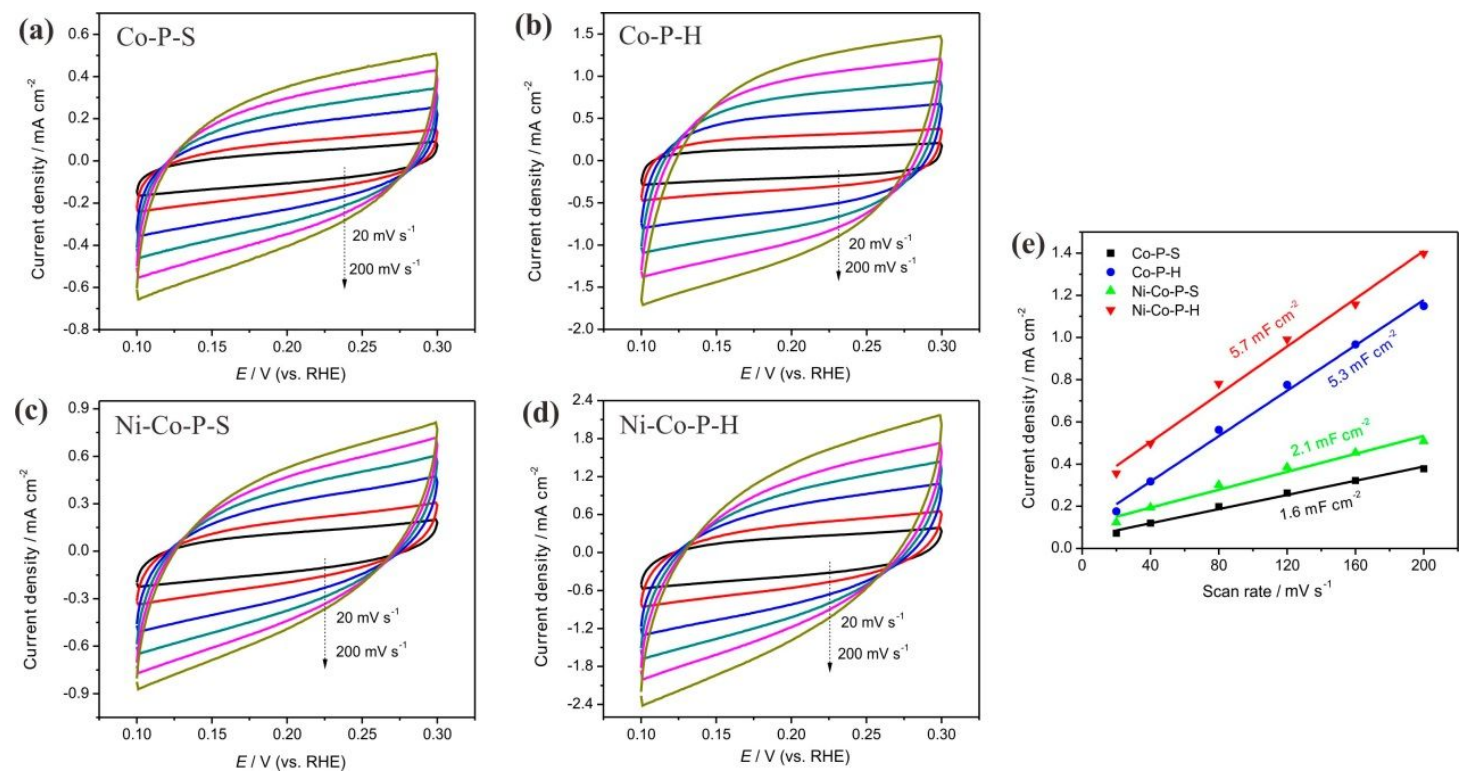

Figure S8 CV curves in the range of 0.1-0.3 V at various scan rates in 0.5 M PBS solution: (a) Co-P-S, (b) Co-P-H, (c) Ni-Co-P-S and (d) Ni-Co-P-H; (e) the calculated capacitive currents depending on a function of scan rate. 

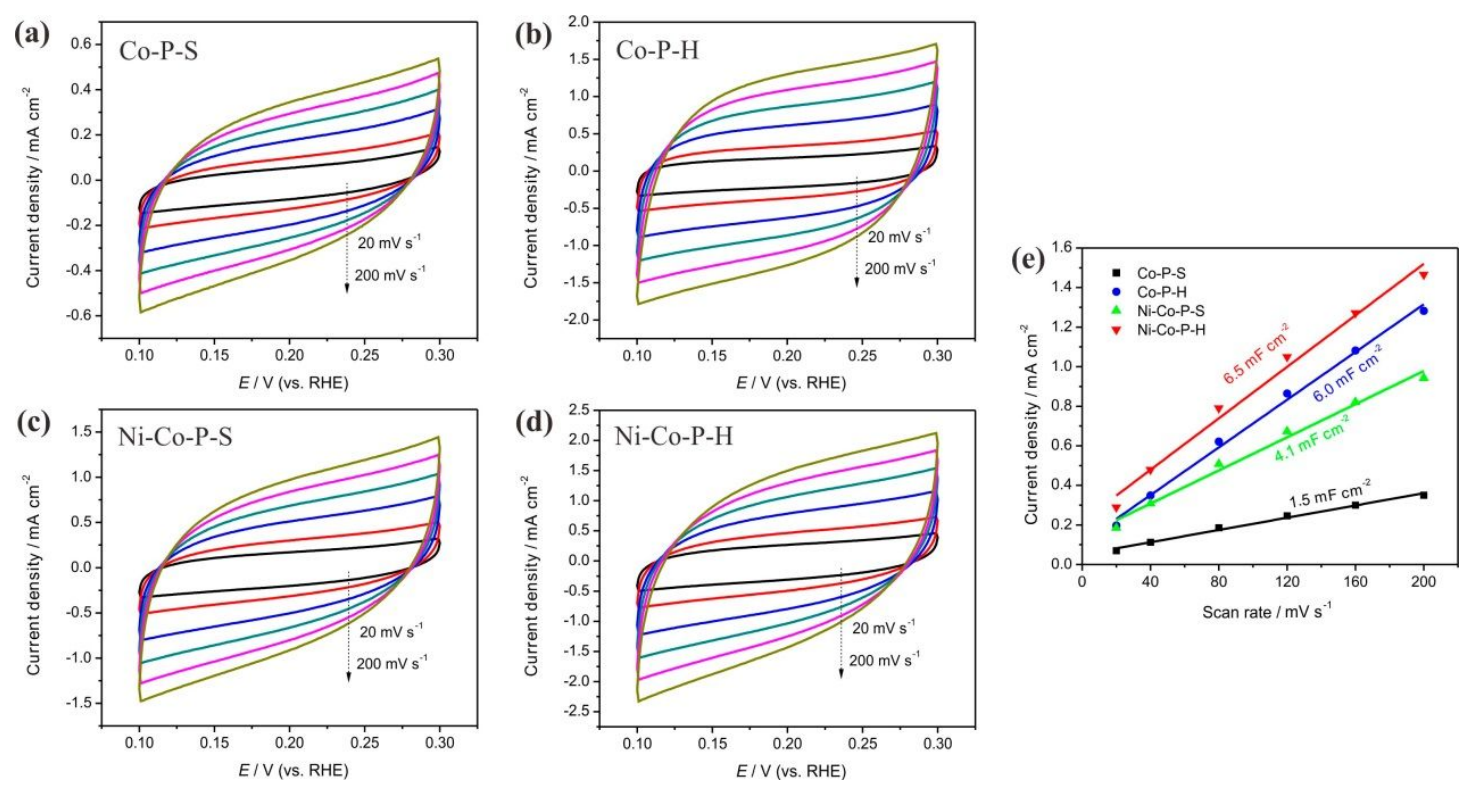

Figure S9 CV curves in the range of $0.1-0.3 \mathrm{~V}$ at various scan rates in $1 \mathrm{M} \mathrm{KOH}$ solution: (a) Co-P-S, (b) Co-P-H, (c) Ni-Co-P-S and (d) Ni-Co-P-H; (e) the calculated capacitive currents depending on a function of scan rate.

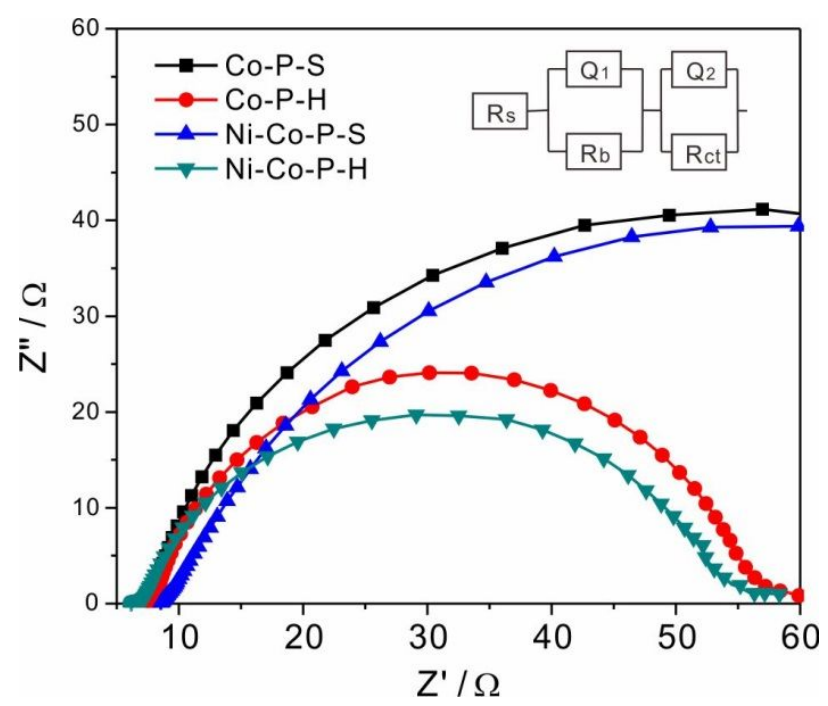

Figure S10 Nyquist plots of Co-P-S, Co-P-H, Ni-Co-P-S and Ni-Co-P-H in $1 \mathrm{M} \mathrm{KOH}$ (inset: the equivalent circuit for Nyquist plots). 
Table S1 Comparison of the HER performances of non-noble metal electrocatalysts in $0.5 \mathrm{M}$ $\mathrm{H}_{2} \mathrm{SO}_{4}$

\begin{tabular}{|c|c|c|c|}
\hline Catalysts & Tafel slope $\left(\mathrm{mV} \mathrm{dec}^{-1}\right)$ & Potential (mV)@10mA cm-2 & References \\
\hline $\mathrm{NiCoP} / \mathrm{CoP}-\mathrm{Ti}_{4} \mathrm{O}_{7}$ & 65.5 & 128 & 2 \\
\hline $\mathrm{Ni}_{2} \mathrm{P}-\mathrm{CoP}$ & 64 & 105 & 3 \\
\hline MoP-RGO-0.5/S 0.01 & --- & 144 & 4 \\
\hline $\mathrm{CoMoS}_{3}$ & 78 & 143 & 5 \\
\hline $\mathrm{N}-\mathrm{Mo}_{2} \mathrm{C} \mathrm{NSs}$ & 44.5 & 99 & 6 \\
\hline $\mathrm{Ni}_{12} \mathrm{P}_{5}$ hollow spheres & 46 & 144 & 7 \\
\hline MoSSe@rGO & 51 & 153 & 8 \\
\hline $\mathrm{Ni}-\mathrm{Co}-\mathrm{P} / \mathrm{C}_{60}$ & 48 & 97 & 9 \\
\hline CoP-60 & 56 & 121 & 10 \\
\hline Ni-Co-P-H & 68 & 90 & This work \\
\hline
\end{tabular}

Table S2 Comparison of the HER performances of non-noble metal electrocatalysts in PBS solution.

\begin{tabular}{lccc}
\hline Catalysts (concentration of PBS) & Tafel slope $\left(\mathrm{mV} \mathrm{dec}^{-1}\right)$ & Potential $(\mathrm{mV}) @ 10 \mathrm{~mA} \mathrm{~cm}^{-2}$ & References \\
\hline CoP NA/CC (1 M) & 123 & 145 & 11 \\
$\mathrm{H}_{2}$-CoCat $(0.5 \mathrm{M})$ & $>385$ & 140 & 12 \\
$\mathrm{CoP}_{3} \mathrm{HSs}(1 \mathrm{M})$ & 102 & 168 & 13 \\
$\mathrm{WP}$ NAs/CC $(1 \mathrm{M})$ & 125 & 200 & 15 \\
$\mathrm{Co}-\mathrm{Fe}-\mathrm{P}(1 \mathrm{M})$ & 138 & 138 & 16 \\
$\mathrm{CoP}-\mathrm{MNA} / \mathrm{NF}(0.5 \mathrm{M})$ & 180 & 189 & 17 \\
$\mathrm{Ni}{ }_{2} \mathrm{P}$ (NPCNFs/CC $(1 \mathrm{M})$ & 230.3 & 185 & This work \\
Ni-Co-P-H $(0.5 \mathrm{M})$ & 84 & 157 & 15 \\
\hline
\end{tabular}


Table S3 Comparison of the HER performances of non-noble metal electrocatalysts in $1 \mathrm{M} \mathrm{KOH}$.

\begin{tabular}{lccc}
\hline \multicolumn{1}{c}{ Catalysts } & Tafel slope $\left(\mathrm{mV} \mathrm{dec}^{-1}\right)$ & Potential $(\mathrm{mV}) @ 10 \mathrm{~mA} \mathrm{~cm}^{-2}$ & References \\
\hline $\mathrm{CoP}(\mathrm{MoP})-\mathrm{CoMoO} @ \mathrm{CN}$ & 95 & 198 & 18 \\
$\mathrm{MoP}-\mathrm{RGO}-0.5 / \mathrm{S}_{0.01}$ & --- & 152 & 4 \\
$\mathrm{~S}, \mathrm{~N}-\mathrm{CNTs} / \mathrm{CoS} 2 @ \mathrm{Co}$ & 104.9 & 112 & 19 \\
$\mathrm{NiCoP}-2.5-300$ & 42 & 124 & 20 \\
$\mathrm{CoP} @ \mathrm{NPCSs}$ & 109 & 115 & 21 \\
$\mathrm{CoMoS}$ & 105 & 133 & 5 \\
$\mathrm{NiCo}$ & $\mathrm{S}_{4} / \mathrm{Ni}$ & 65 & 22 \\
$\mathrm{CoP} \mathrm{Nanosheet} \mathrm{Aerogel}$ & 72 & 154 & 23 \\
$\mathrm{Ni}_{0.69} \mathrm{Co}_{0.31}-\mathrm{P}$ & 47 & 96 & 24 \\
$\mathrm{Ni}^{-\mathrm{Co}-\mathrm{P}-\mathrm{H}}$ & 65 & 121 & This work \\
\hline
\end{tabular}




\section{References}

(1) Tan, C.; Cao, X.; Wu, X. J.; He, Q.; Yang, J.; Zhang, X.; Chen, J.; Zhao, W.; Han, S.; Nam, G. H.; Sindoro, M.; Zhang, H. Recent Advances in Ultrathin Two-Dimensional Nanomaterials. Chem. Rev. 2017, 117, 6225-6331.

(2) Ma, D.; Li, R.; Zheng, Z.; Jia, Z.; Meng, K.; Wang, Y.; Zhu, G.; Zhang, H.; Qi, T. Nicop/Cop Nanoparticles Supported on $\mathrm{Ti} 4 \mathrm{o} 7$ as the Electrocatalyst Possessing an Excellent Catalytic Performance toward the Hydrogen Evolution Reaction. ACS Sustain. Chem. Eng. 2018, 6, $14275-14282$.

(3) Liang, X.; Zheng, B.; Chen, L.; Zhang, J.; Zhuang, Z.; Chen, B. Mof-Derived Formation of Ni2p-Cop Bimetallic Phosphides with Strong Interfacial Effect toward Electrocatalytic Water Splitting. ACS Appl. Mater. Interfaces 2017, 9, 23222-23229.

(4) Wu, Z.; Song, M.; Zhang, Z.; Wang, J.; Liu, X. Various Strategies to Tune the Electrocatalytic Performance of Molybdenum Phosphide Supported on Reduced Graphene Oxide for Hydrogen Evolution Reaction. J. Colloid Interface Sci. 2019, 536, 638-645.

(5) Guo, J.; Zhang, X.; Sun, Y.; Tang, L.; Zhang, X. Self-Template Synthesis of Hierarchical Comos3 Nanotubes Constructed of Ultrathin Nanosheets for Robust Water Electrolysis. J. Mater. Chem. A 2017, 5, 11309-11315.

(6) Jia, J.; Xiong, T.; Zhao, L.; Wang, F.; Liu, H.; Hu, R.; Zhou, J.; Zhou, W.; Chen, S. Ultrathin N-Doped Mo2c Nanosheets with Exposed Active Sites as Efficient Electrocatalyst for Hydrogen Evolution Reactions. ACS nano 2017, 11, 12509-12518.

(7) Chang, J.; Li, S.; Li, G.; Ge, J.; Liu, C.; Xing, W. Monocrystalline Ni12p5 Hollow Spheres with Ultrahigh Specific Surface Areas as Advanced Electrocatalysts for the Hydrogen Evolution Reaction. J. Mater. Chem. A 2016, 4, 9755-9759.

(8) Konkena, B.; Masa, J.; Xia, W.; Muhler, M.; Schuhmann, W. Mosse@Reduced Graphene Oxide Nanocomposite Heterostructures as Efficient and Stable Electrocatalysts for the Hydrogen Evolution Reaction. Nano Energy 2016, 29, 46-53.

(9) Du, Z.; Jannatun, N.; Yu, D.; Ren, J.; Huang, W.; Lu, X. C60-Decorated Nickel-Cobalt Phosphide as an Efficient and Robust Electrocatalyst for Hydrogen Evolution Reaction. Nanoscale 2018, 10, 23070-23079.

(10) Guo, X.; Liang, J.; Wang, L.; Feng, Z.; Yu, T.; Zhang, Z.; Shao, Y.; Hao, C.; Li, G. Synthesis of Cobalte-Glycerate Hierarchical Structure and Their Conversion into Hierarchical Cop Nanospheres for the Hydrogen Evolution Reaction. Inter. J. Hydrogen Energ. 2018, 43, 2034-2042.

(11) Liu, T.; Xie, L.; Yang, J.; Kong, R.; Du, G.; Asiri, A. M.; Sun, X.; Chen, L. Self-Standing Cop Nanosheets Array: A Three-Dimensional Bifunctional Catalyst Electrode for Overall Water Splitting in Both Neutral and Alkaline Media. ChemElectroChem 2017, 4, 1840-1845.

(12) Cobo, S.; Heidkamp, J.; Jacques, P. A.; Fize, J.; Fourmond, V.; Guetaz, L.; Jousselme, B.; Ivanova, V.; Dau, H.; Palacin, S.; Fontecave, M.; Artero, V. A Janus Cobalt-Based Catalytic Material for Electro-Splitting of Water. Nat. Mater. 2012, 11, 802-7.

(13) Wu, T.; Pi, M.; Wang, X.; Guo, W.; Zhang, D.; Chen, S. Hierarchical Cobalt Poly-Phosphide Hollow Spheres as Highly Active and Stable Electrocatalysts for Hydrogen Evolution over a Wide $\mathrm{Ph}$ Range. Appl. Surf. Sci. 2018, 427, 800-806.

(14) Pu, Z.; Liu, Q.; Asiri, A. M.; Sun, X. Tungsten Phosphide Nanorod Arrays Directly Grown on Carbon Cloth: A Highly Efficient and Stable Hydrogen Evolution Cathode at All Ph Values. ACS Appl. Mater. Interfaces 2014, 6, 21874-9. 
(15) Chen, J.; Liu, J.; Xie, J.; Ye, H.; Fu, X.; Sun, R.; Wong, C. Co-Fe-P Nanotubes Electrocatalysts Derived from Metal-Organic Frameworks for Efficient Hydrogen Evolution Reaction under Wide Ph Range. Nano Energy 2019, 56, 225-233.

(16) Zhu, Y.; Liu, Y.; Ren, T.; Yuan, Z. Self-Supported Cobalt Phosphide Mesoporous Nanorod Arrays: A Flexible and Bifunctional Electrode for Highly Active Electrocatalytic Water Reduction and Oxidation. Adv. Fun. Mater. 2015, 25, 7337-7347.

(17) Wang, M. Q.; Ye, C.; Liu, H.; Xu, M.; Bao, S. J. Nanosized Metal Phosphides Embedded in Nitrogen-Doped Porous Carbon Nanofibers for Enhanced Hydrogen Evolution at All Ph Values. Angew. Chem. Int. Ed. 2018, 57, 1963-1967.

(18) Yu, L.; Xiao, Y.; Luan, C.; Yang, J.; Qiao, H.; Wang, Y.; Zhang, X.; Dai, X.; Yang, Y.; Zhao, H. Cobalt/Molybdenum Phosphide and Oxide Heterostructures Encapsulated in N-Doped Carbon Nanocomposite for Overall Water Splitting in Alkaline Media. ACS Appl. Mater. Interfaces 2019, 11, 6890-6899.

(19) Wang, J.-Y.; Ouyang, T.; Li, N.; Ma, T.; Liu, Z.-Q. S, N Co-Doped Carbon Nanotube-Encapsulated Core-Shelled Cos2@Co Nanoparticles: Efficient and Stable Bifunctional Catalysts for Overall Water Splitting. Sci. Bull. 2018, 63, 1130-1140.

(20) Li, Y.; Liu, J.; Chen, C.; Zhang, X.; Chen, J. Preparation of Nicop Hollow Quasi-Polyhedra and Their Electrocatalytic Properties for Hydrogen Evolution in Alkaline Solution. ACS Appl. Mater. Interfaces 2017, 9, 5982-5991.

(21) Wu, K.; Chen, Z.; Cheong, W. C.; Liu, S.; Zhu, W.; Cao, X.; Sun, K.; Lin, Y.; Zheng, L.; Yan, W.; Pan, Y.; Wang, D.; Peng, Q.; Chen, C.; Li, Y. Toward Bifunctional Overall Water Splitting Electrocatalyst: General Preparation of Transition Metal Phosphide Nanoparticles Decorated N-Doped Porous Carbon Spheres. ACS Appl. Mater. Interfaces 2018, 10, 44201-44208.

(22) Ma, L.; Hu, Y.; Chen, R.; Zhu, G.; Chen, T.; Lv, H.; Wang, Y.; Liang, J.; Liu, H.; Yan, C.; Zhu, H.; Tie, Z.; Jin, Z.; Liu, J. Self-Assembled Ultrathin Nico 2 S 4 Nanoflakes Grown on Ni Foam as High-Performance Flexible Electrodes for Hydrogen Evolution Reaction in Alkaline Solution. Nano Energy 2016, 24, 139-147.

(23) Li, H.; Zhao, X.; Liu, H.; Chen, S.; Yang, X.; Lv, C.; Zhang, H.; She, X.; Yang, D. Sub-1.5 Nm Ultrathin Cop Nanosheet Aerogel: Efficient Electrocatalyst for Hydrogen Evolution Reaction at All Ph Values. Small 2018, 1802824.

(24) Yin, Z.; Zhu, C.; Li, C.; Zhang, S.; Zhang, X.; Chen, Y. Hierarchical Nickel-Cobalt Phosphide Yolk-Shell Spheres as Highly Active and Stable Bifunctional Electrocatalysts for Overall Water Splitting. Nanoscale 2016, 8, 19129-19138. 